\title{
MORBILIDAD MATERNA EXTREMA EN LA UNIDAD DE CUIDADOS INTENSIVOS DE UN HOSPITAL DE HUANCAVELICA
}

\section{EXTREME MATERNAL MORBIDITY IN THE INTENSIVE CARE UNIT OF A HUANCAVELICA HOSPITAL, PERU}

\author{
Martiza Jorge-Chahuayo (iD 1,a, Elizabeth Vilca-Aponte (iD) 1,b, Enma Jaurapoma-Lizana (iD) 1,c
}

\begin{abstract}
Objetivo: Determinar las características personales, obstétricas y clínicas de las pacientes que presentaron morbilidad materna extrema. Material y métodos: Estudio descriptivo, retrospectivo de corte transversal, realizado en el Hospital Departamental de Huancavelica (Perú), donde se revisaron 94 historias clínicas de gestantes o puérperas atendidas en Unidad de Cuidados Intensivos (UCl) entre enero 2016 a diciembre 2018. Resultados: Las características personales de las pacientes con morbilidad materna extrema fueron; presentan una edad materna entre 20 a 34 años $(53,2 \%)$, estado civil soltera $(35,1 \%)$, grado de instrucción secundario $(45,7 \%)$ y procedencia rural $(63,8 \%)$. Respecto de las características obstétricas, presentaron una paridad de nulíparas y multíparas $(26,6 \%), \geq 6$ controles prenatales $(59,6 \%)$ y un periodo intergenésico adecuado $(67,0 \%)$. Finalmente, las características clínicas más resaltantes de esta población fueron presentar preeclampsia severa $(48,9 \%)$, shock hipovolémico (13.8\%), síndrome de Hellp (12.8\%), eclampsia (11.7\%), aborto (5.3\%), hemorragia posparto (3.2\%) y shock séptico (3.2\%). Conclusión: En mujeres con morbilidad materna extrema, más de la mitad tienen edades entre 20 a 34 años, la mayoría solteras; nivel secundario y proceden del área rural, nulíparas y multigestas, con más de 6 controles prenatales y tienen periodo intergenésico adecuado, finalmente la preeclampsia severa fue más frecuente.
\end{abstract}

Palabras clave: Morbilidad, Salud materna, Unidad de cuidados intensivos, Características de la población (Fuente: DeCS, BIREME)

Objective: To determine the personal, obstetric, and clinical characteristics of patients who had extreme maternal morbidity. Material and methods: Descriptive, cross-sectional retrospective study, conducted at the Departmental Hospital of Huancavelica (Peru), where 94 medical histories of pregnant or postpartums attended in Intensive Care Unit (ICU) were reviewed between January 2016 and December 2018. Results: The personal characteristics of patients with extreme maternal morbidity where are 20 to 34 years old (53.2\%), single marital status (35.1\%), secondary education (45.7\%) and rural origin (63.8\%). Regarding obstetric characteristics, they had a parity of nulliparates and multiparas (26.6\%), 6 prenatal controls (59.6\%) and an adequate intergeny period (67.0\%). Finally, the clinical characteristics of this population were characterized by severe preeclampsia (48.9\%), hypovolemic shock (13.8\%), Hellp syndrome (12.8\%), eclampsia (11.7\%), abortion (5.3\%), postpartum hemorrhage (3.2\%) and septic shock (3.2\%). Conclusion: In women with extreme maternal morbidity, more than half are between 20 and 34 years old, most of them single; secondary level and come from rural, Nulipara and multigestas, with more than 6 prenatal checkups and have adequate intergenetic period, finally severe preeclampsia was more frequent.

Key words: Mordibity, Maternal health, Intensive care unit, Characteristics of the population (Source: MeSH NLM)

Filiación:

1. Facultad de Enfermería, Universidad Nacional de Huancavelica. Huancavelica, Perú.

. Magíster en Gestión de los Servicios de Salud. Obstetra. b. Especialista en Centro Quirúrgico Especializado. Licenciada en Enfermería.

c. Magíster en Administración y Gerencia de los Servicios

de Salud. Licenciada en Enfermería.
Citar como: Jorge-Chahuyo $\mathrm{M}$, Vilca-Aponte E, Jaurapoma-Lizana E. Revista Internacional de Salud Materno Fetal. 2020; 5 (2): 18-23. DOI:

https://doi.org/10.47784/rismf .2020.5.2.83
Financiamiento: Autofinanciado por los autores

Conflictos de interés: Los autores declaran no presentar conflictos de interés.

Correspondencia: Martiza JorgeChahuayo (maritajcha2219@gmail.com)

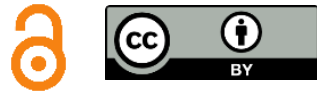

Recibido: 08 de Junio del 2020 Aprobado: 22 de Junio del 2020 Publicado: 30 de Junio del 2020 


\section{INTRODUCCIÓN}

La Federación Latinoamericana de Sociedades de Ginecobstetricia (FLASOG) y la Organización Mundial de la Salud (OMS) definen la MME como "una complicación severa que ocurre durante el embarazo, parto o dentro de los 42 días posteriores a la terminación del embarazo, pero sobrevive gracias a una intervención médica urgente y oportuna" (1). La MME constituye un indicador de los cuidados obstétricos y uno de los temas que más atención ha recibido a nivel mundial por su estrecha relación con la mortalidad materna reconociéndose que por cada muerte de una paciente obstétrica cerca de 118 mujeres sufren una MME (2). En este sentido, la Organización Mundial de la Salud (OMS), al igual que distintos organismos internacionales en el campo de la salud, en las últimas décadas ha demostrado interés en el estudio de los problemas de la mujer y en la disminución de la mortalidad materna (3-4).

En América Latina, existen pocos reportes acerca del tema, desconociéndose la magnitud real del problema (5); Sin embargo, la Organización Mundial de la Salud calcula que, por cada muerte materna, 135 mujeres presentan enfermedades relacionadas con la gestación y aproximadamente 30 quedan con secuelas. La Federación Latinoamericana de Sociedades de Obstetricia y Ginecología (FLASOG) publicó 965 casos expuestos en el estudio titulado "Caracterización de la morbilidad materna extremadamente grave en instituciones seleccionadas de América Latina", realizado en el periodo del 1 de junio de 2007 al 31 de mayo de 2008, donde se identificó como primera causa de morbilidad materna a los trastornos hipertensivos con $44,3 \%$, seguido de la hemorragia posparto con 17,9\% (6). Así mismo un estudio realizado entre marzo y junio de 2013 en 19 hospitales de 12 países de América Latina encontró una incidencia de maternal near miss de 12,9 casos por 1.000 nacidos vivos. La relación $\mathrm{morbilidad} / \mathrm{mortalidad}$ fue de 19 a 1 con un índice de mortalidad del $5,1 \%(7)$.

En nuestro país, además de la información de la mortalidad materna, el análisis de la morbilidad materna extrema, que es complemento necesario para evaluar las necesidades y definir prioridades, aún está en su fase inicial, no existiendo estudios en nuestro país, ni en los hospitales de la capital. A pesar de la relativamente alta razón de mortalidad de nuestro país, el número absoluto de muertes institucionales es bajo; por lo tanto, se requiere un indicador de calidad de atención diferente a la mortalidad (8).

Así mismo en la región Huancavelica, no existen estudios referentes a la morbilidad materna extrema, siendo los casos de muerte materna los más estudiados, siendo así que más del $80 \%$ de las muertes maternas pueden ser evitadas, sin embargo, los casos morbilidad materna extrema (MME) se incrementaron, teniendo 99 casos de MME para el año 2016, 87 casos en el 2017 y 102 casos en el 2018, motivo por el cual también se realiza el presente estudio de investigación (9-10).

En este sentido el presente estudio tuvo el propósito de determinar las características de las mujeres con morbilidad materna extrema, cuyos resultados permitirán fortalecer las capacidades del personal de salud en el primer y segundo nivel de atención así mismo tomar acciones necesarias para mejorar la calidad de atención de las usuarias

\section{MATERIAL Y MÉTODOS}

\section{TIPO DE ESTUDIO}

El presente estudio es observacional, descriptivo, longitudinal retrospectivo.

\section{POBLACIÓN}

Constituida por 94 pacientes obstétricas que ingresaron a la Unidad de Cuidados Intensivos (UCl) del Hospital Departamental de Huancavelica, en Perú, durante los años 2016 al 2018, establecimiento de salud categoría II-2 y, atiende a población afiliada al Seguro Integral de Salud (SIS), un seguro gratuito subsidiado por el Estado.

En este contexto la muestra fue censal; porque se trabajó con toda la población; es decir con todas las pacientes obstétricas que ingresaron a la Unidad de Cuidados Intensivos, del mismo modo en los criterios de inclusión y exclusión se consideró las historias clínicas de todas las pacientes que ingresaron a la $\mathrm{UCl}$ y se excluyeron las historias clínicas incompletas.

\section{VARIABLES}

Se midieron las características personales: Edad (años), estado civil, grado de instrucción y 
procedencia. Para las características obstétricas se midieron la paridad, control prenatal y periodo intergenésico (años) y para las características clínicas: Shock hipovolémico, hemorragia postparto, eclampsia, preeclampsia severa, síndrome de Hellp, shock séptico, sepsis y aborto.

\section{TÉCNICAS E INSTRUMENTOS}

Se empleó el análisis documental, el instrumento fue la ficha de recolección, cual fue llenado con datos obtenidos de las historias clínicas de la población de estudio.

\section{PROCEDIMIENTOS}

El proceso de recolección de datos se realizó durante el año 2019, para lo cual se solicitó la autorización institucional, en coordinación con la Unidad de Estadística e Informática, para que nos facilite las historias clínicas de las pacientes, según la base de datos que nos brindó el servicio de UCl.

\section{ANÁLISIS DE DATOS}

Para el análisis de datos se elaboró una base de datos en una hoja de cálculo en Microsoft Office Excel 2016; esto se realizó por el fácil uso y familiaridad con el aplicativo ofimático. Para el análisis estadístico se importó la base de datos del Excel al programa estadístico SPSS Statistics para Windows (versión 25.0), con la finalidad de realizar el análisis estadístico descriptivo según la naturaleza del estudio. Se utilizaron medidas descriptivas para reportar los resultados, generando frecuencias y porcentajes de cada una de las variables seleccionadas.

\section{ASPECTOS ÉTICOS}

El estudio fue aprobado por el Comité de ética e investigación del Hospital Departamental de Huancavelica, el 13 de noviembre del 2019 con Carta NNo 052 - 2019/GOB.REG-HVCA/HRZCV-HVCA/OADI.

\section{RESULTADOS}

El $53,2 \%$ de mujeres con morbilidad materna extrema tienen edades entre 20 a 34 años; el 35,1\% son solteras; el $45,7 \%$ tienen grado de instrucción el nivel secundario y el $63,8 \%$ de mujeres con morbilidad materna extrema proceden del área rural (Tabla 1).
Tabla 1. Características personales de las mujeres con morbilidad materna extrema atendidas en la unidad de cuidados intensivos del Hospital Departamental de Huancavelica, 2016 a 2018

\begin{tabular}{lcc}
\hline & $\mathbf{n}$ & $\%$ \\
\hline Edad & 1 & \\
$\leq 15$ años & 22 & 1.1 \\
$16-19$ años & 50 & 23.4 \\
$20-34$ años & 21 & 53.2 \\
$\geq 35$ años & & \\
Estado civil & 29 & 30.9 \\
Casada & 31 & 33.0 \\
Conviviente & 33 & 35.1 \\
Soltera & 1 & 1.1 \\
Otro & & \\
Grado de instrucción & 13 & 13.8 \\
Sin estudios & 8 & 8.5 \\
Primaria & 43 & 45.7 \\
Secundaria & 13 & 13.8 \\
Superior no universitaria & 17 & 18.1 \\
Superior universitaria & & \\
Procedencia & 60 & 63.8 \\
Rural & 34 & 36.2 \\
Urbana & 94 & 100,0 \\
\hline
\end{tabular}

El $26,6 \%$ de mujeres con morbilidad materna extrema son nulíparas y multíparas respectivamente; el $59,6 \%$ de mujeres con morbilidad materna extrema tienen más de 6 controles prenatales; y el $67,9 \%$ de mujeres con morbilidad materna extrema tienen un periodo intergenésico adecuado de 2 a 5 años (Tabla 2).

Tabla 2. Características obstétricas de las mujeres con morbilidad materna extrema atendidas en la unidad de cuidados intensivos del Hospital Departamental de Huancavelica, 2016 a 2018

\begin{tabular}{|c|c|c|}
\hline & $\mathrm{n}$ & $\%$ \\
\hline \multicolumn{3}{|l|}{ Paridad } \\
\hline Nulípara & 25 & 26.6 \\
\hline Primípara & 22 & 23.4 \\
\hline Multípara & 25 & 26.6 \\
\hline Gran multípara & 22 & 23.4 \\
\hline \multicolumn{3}{|l|}{ Control prenatal (CPN) } \\
\hline$<6 \mathrm{CPN}$ & 18 & 19.1 \\
\hline$\geq 6 \mathrm{CPN}$ & 56 & 59.6 \\
\hline Sin CPN & 20 & 21.3 \\
\hline \multicolumn{3}{|l|}{ Periodo intergenésico } \\
\hline Corto (< a 2 años) & 20 & 21.3 \\
\hline Adecuado (2-5 años) & 63 & 67.0 \\
\hline Largo (> 5 años) & 11 & 11.7 \\
\hline Total & 94 & 100,0 \\
\hline
\end{tabular}


Del total de mujeres con morbilidad materna extrema el 48,9\% presentan preeclampsia severa, $13,8 \%$ shock hipovolémico, 12,8\% síndrome de Hellp y otras características clínicas en menor número. (Tabla 3).

Tabla 3. Características clínicas según presencia de morbilidad materna extrema en la unidad de cuidados intensivos del Hospital Departamental de Huancavelica, 2016 a 2018

\begin{tabular}{|c|c|c|c|c|c|c|}
\hline & \multicolumn{6}{|c|}{ Morbilidad materna extrema } \\
\hline & \multicolumn{2}{|c|}{$\mathrm{Si}$} & \multicolumn{2}{|c|}{ No } & \multicolumn{2}{|c|}{ Total } \\
\hline & $\mathrm{n}$ & $\%$ & $\mathrm{n}$ & $\%$ & $\mathrm{n}$ & $\%$ \\
\hline $\begin{array}{l}\text { Shock } \\
\text { hipovolémico }\end{array}$ & 13 & 13.8 & 81 & 86.2 & 94 & 100 \\
\hline $\begin{array}{l}\text { Hemorragia } \\
\text { postparto }\end{array}$ & 3 & 3.2 & 91 & 96.8 & 94 & 100 \\
\hline Eclampsia & 11 & 11.7 & 83 & 88.3 & 94 & 100 \\
\hline $\begin{array}{l}\text { Preeclampsia } \\
\text { severa }\end{array}$ & 46 & 48.9 & 48 & 51.1 & 94 & 100 \\
\hline $\begin{array}{l}\text { Síndrome de } \\
\text { Hellp }\end{array}$ & 12 & 12.8 & 82 & 87.2 & 94 & 100 \\
\hline Shock séptico & 3 & 3.2 & 91 & 96.8 & 94 & 100 \\
\hline Sepsis & 0 & 0.0 & 94 & 100.0 & 94 & 100 \\
\hline Aborto & 5 & 5.3 & 89 & 94.7 & 94 & 100 \\
\hline
\end{tabular}

\section{DISCUSIÓN}

La morbilidad materna extremadamente grave, es un indicador asociado a la muerte materna y es considerada una alternativa muy importante para utilizarla como indicador de la calidad de los cuidados maternos en los establecimientos de salud.

En este estudio se describieron las características personales donde se encontró que del total de mujeres con morbilidad materna extrema el 53,2\% son mujeres de entre 20 a 34 años; estos resultados coinciden con lo obtenido por Álvarez (11) en el que la edad promedio de las mujeres con morbilidad materna extrema es de 28 años, sin embargo, estos resultados difieren de los hallados por Reyes (12), que determina la morbilidad materna extrema estuvo relacionado con la edad materna mayor a 35 años. Del mismo modo se obtuvo como resultado que del total de mujeres el $35,1 \%$ son solteras, lo que a diferencia con lo encontrado por Bendezú (13) que refiere que del total el $48 \%$ eran convivientes las que presentaron morbilidad materna. En el mismo contexto se evidencia que el $45,7 \%$ de mujeres que presentaron morbilidad materna extrema tienen como grado de instrucción el nivel secundario, lo que no concuerda por lo encontrado por Reyes (12) quien refiere que la morbilidad materna extrema se presentó en mujeres con bajo nivel educativo. Finalmente, el 63,8\% del total de mujeres con morbilidad materna extrema proceden del área rural lo que coincide con los resultados obtenidos por Chagua (14) que refiere que existe relación significativa entre procedencia con la morbilidad materna extrema.

En cuanto a las características obstétricas, se observa que del total el $59,6 \%$ son multíparas y nulíparas respectivamente, lo que coincide con lo encontrado por Reyes (12) y Chagua (14) que refieren que la morbilidad materna extrema estuvo relacionada con la multiparidad y nuliparidad respectivamente. De la misma forma del total de mujeres con morbilidad materna extrema el 59,6\% tienen más de 6 controles prenatales lo que coincide con lo encontrado por Chagua (14) que refiere que el $62.2 \%$ de mujeres con morbilidad materna extrema tuvieron más de 6 atenciones prenatales. Finalmente, con respecto al periodo intergenésico del total de mujeres con morbilidad materna extrema el 67,9\%; tienen un periodo intergenésico adecuado de 2 a 5 años, lo que coincide con lo referido por la Organización Mundial de la Salud (OMS), sin embargo, no contrasta con los resultados encontrados por Reyes (12) que refiere que la morbilidad materna extrema estuvo relacionada con periodos intergenésicos cortos o prolongados.

Según las características clínicas de las mujeres con morbilidad materna extrema se observó que el $48,9 \%$ del total presentan preeclampsia severa, y otras características clínicas en menor número, estos resultados contrastan con lo encontrado por Chagua (14) que manifiesta que el $78,9 \%$ de las mujeres presentaron preeclampsia como complicación obstétrica de la morbilidad materna extrema, así mismo Reyes (12) refiere que la enfermedad hipertensiva de la gestación fue la causa más importante de morbilidad materna; sin embargo estos resultados no contrastan con lo referido por Castañeda (15) que encontró como resultado que la hemorragia obstétrica es la principal causa de morbilidad materna.

Ante los resultados encontrados en el estudio se recomienda a la Dirección Regional de Salud de Huancavelica; el fortalecimiento de competencias del personal de salud del primer nivel de atención en la 
atención prenatal adecuada y el diagnóstico oportuno de la preeclampsia, así mismo fortalecer el seguimiento a las gestantes. Así mismo durante el desarrollo del estudio no se tuvo limitaciones y fue factible culminar toda vez que se contó con recurso económico, logístico, tiempo.

Finalmente se encontró que las mujeres que presentaron morbilidad materna extrema tuvieron más de 6 controles prenatales, de procedencia rural y la morbilidad más frecuente fue la preeclampsia severa. 


\section{REFERENCIAS}

1. Acelas-Granados David F, Orostegui Andrea, Alarcón-Nivia Miguel Ángel. Factores de riesgo para morbilidad materna extrema en gestantes sin demora en la atención médica según la estrategia camino para la supervivencia. Rev. chil. obstet. ginecol. [Internet]. 2016 Jun [citado 2020 Jun 14]; 81 (3): 181188. [Link]

2. Hernández-Oliva $M$, Padrón-Mora $M$, Díaz-García J. Factores de riesgo de morbilidad y mortalidad materna en una unidad de cuidados intensivos / Morbidity and mortality risk factors in critically ill pregnant women in an intensive care unit. Revista Cubana de Medicina Intensiva y Emergencias [revista en Internet]. 2017 [citado 2020 Jun 7]; 16(3):1. [Link]

3. Starrs, Ann. 1987. Preventing the tragedy of maternal deaths: a report on the International Safe Motherhood Conference (English). Washington, D.C.: World Bank Group. [Link]

4. Organización Mundial de la Salud (OMS). Más allá de las cifras, revisión de las muertes maternas y las complicaciones del embarazo para hacer la maternidad segura [Internet]. Centro Latinoamericano de Perinatología, salud de la mujer y reproductiva. 2004 [Citado el 2 de marzo de 2017]. [Link]

5. Organización Mundial de la Salud. Ouagadougou Declaration on Primary Health Care and Health Systems in Africa. In Achieving better health for Africa in the New Millennium; 2008; Ginebra.
6. Rojas JA, Cogollo M, Miranda JE, Ramos EC, Fernández JC, Bello AM. Morbilidad materna extrema en cuidados intensivos obstétricos. Cartagena (Colombia) 2006 - 2008. Rev. Colomb. Obstet. Ginecol. [Internet]. 30 de junio de 2011 [citado 14 de junio de 2020];62(2):131-40. [Link]

7. Mejia M, Aura M, Téllez-Becerril GE, González-Vargas Á. Morbilidad materna extrema (near miss) y muertes maternas. Archivos de Investigación Materno Infantil. 2012 diciembre; 4(3).

8. Calvo-Aguilar O, Morales-García VE, Fabián-Fabián J. Morbilidad materna extrema en el Hospital General Dr. Aurelio Valdivieso, Servicios de Salud de Oaxaca. Ginecol Obstet Mex. 2010;78(12):660-668. [Link]

9. Soni-Trinidad C, Gutiérrez-Mateos A Santa Rosa-Moreno FJ, et al. Morbilidad y mortalidad materna y factores de riesgo asociados con una urgencia obstétrica. Ginecol Obstet Mex. 2015;83(02):96-103. [Link]

10. Oficina General de Epidemiología de la Dirección Regional de Salud Huancavelica 2018. Documento técnico.
11. Álvarez Toste Mireya, Salvador Álvarez Sergio, González Rodríguez Guillermo, Pérez Disnardo Raúl. Caracterización de la morbilidad materna extremadamente grave. Rev Cubana Hig Epidemiol [Internet]. 2010 Dic [citado 2020 Jun 07];48(3):310-320. [Link]

12. Reyes-Armas Iván, Villar Aurora. Morbilidad materna extrema en el Hospital Nacional Docente Madre-Niño San Bartolomé, Lima, 2007-2009. Rev. peru. ginecol. obstet. [Internet]. 2012 [citado 2020 Jun 07]; 58 (4): 273-284 [Link]

13. Bendezú Guido, Bendezú-Quispe Guido. Caracterización de la morbilidad materna extremadamente grave en un hospital del seguro social del Perú. Rev. Peru Ginecol Obstet. [Internet]. 2014 Oct [citado 2020 Jun 07]; 60 (4):291-298. [Link]

14. Chagua Pariona, Oscar Luis. Factores de Riesgo asociados a morbilidad materna extrema en el Hospital Regional de Huancavelica. 2017. Tesis para optar el grado de maestría.

15. Castañeda Barberán Denia, Martín Díaz Geidy, Estévez Reinó Arnaldo, Álvarez Toste Mireya, Salvador Álvarez Sergio. Caracterización de la morbilidad materna extremadamente grave en la provincia de Camagüey (2009). Rev Cubana Hig Epidemiol [Internet]. 2014 Ago [citado 2020 Jun 07]; 52(2): 152 162. [Link] 